\title{
Diagonal Circuit Identity Testing and Lower BOUNDS
}

\author{
Nitin SAXENA \\ Centrum voor Wiskunde en Informatica \\ Amsterdam, The Netherlands \\ ns@cwi.nl
}

October 19, 2007

\section{Abstract of Dagstuhl Talk}

In this talk we give a deterministic polynomial time algorithm for testing whether a diagonal depth-3 circuit $C\left(x_{1}, \ldots, x_{n}\right)$ (i.e. $C$ is a sum of powers of linear functions) is zero. We also prove an exponential lower bound showing that such a circuit will compute determinant or permanent only if there are exponentially many linear functions. Our techniques generalize to the following results:

1. Suppose we are given a depth-3 circuit of the form:

$$
C\left(x_{1}, \ldots, x_{n}\right):=\sum_{i=1}^{k} \ell_{i, 1}^{e_{i, 1}} \cdots \ell_{i, s}^{e_{i, s}}
$$

where, $\ell_{i, j}$ 's are linear functions living in $\mathbb{F}\left[x_{1}, \ldots, x_{n}\right]$. We can test whether $C$ is zero in deterministic time poly $\left(n k, \max \left\{\left(1+e_{i, 1}\right) \cdots\left(1+e_{i, s}\right) \mid 1 \leqslant i \leqslant k\right\}\right)$. This immediately gives a deterministic poly $\left(n k 2^{d}\right)$ time identity test for general depth-3 circuits of degree $d$.

2. We prove that if the above circuit $C\left(x_{1}, \ldots, x_{n}\right)$ with a "small" $s=o\left(\frac{m}{\log m}\right)$ computes the determinant (or permanent) of an $m \times m$ matrix then $k=2^{\Omega(m)}$.

Our results work for all fields $\mathbb{F}$. (Previous exponential lower bounds for depth-3 only work for nonzero characteristic.)

Dagstuhl Seminar Proceedings 07411

Algebraic Methods in Computational Complexity

http://drops.dagstuhl.de/opus/volltexte/2008/1308 